\title{
Syntaxe des incises de citation
}

\author{
Olivier Bonami \\ Université Paris-Sorbonne et Laboratoire de Linguistique Formelle (UMR 7110) \\ olivier.bonami@paris-sorbonne.fr \\ Danièle Godard \\ Laboratoire de Linguistique Formelle (UMR 7110 - CNRS \& U. Paris 7)
}

\section{Introduction}

Les incises de citation sont illustrées en (1). Elles sont à comparer avec les autres incises (2), et avec les constructions du discours rapporté (3), avec lesquelles elles partagent certaines propriétés.

(1) a. «Le Président, annonce Marie, est arrivé. »

b. « Marie était très joyeuse », se souvenait Paul.

(2) a. Marie, paraît-il, viendra ce soir.

b. Le président arrivera, son porte-parole l'a annoncé, avec une heure de retard.

(3) a. Alors, il annonce : « Le président est arrivé. »

b. Alors, il annonce que le « président » est arrivé.

c. Alors, il annonce que le président est arrivé.

d. Il s'interrogeait : que répondre à son père ?

Il n'y a pas d'accord dans la littérature sur la définition d'une « citation ». Selon les auteurs, on appelle ainsi :

- le discours direct (3a);

- toutes les expressions en mention, dont l'utilisation représente le choix d'un agent autre que le locuteur $(3 \mathrm{a}, 3 \mathrm{~b})$, et qui sont typiquement signalées par des guillemets ou par une prosodie particulière ;

- $\quad$ tout contenu attribuable à un agent autre que le locuteur, y compris le discours indirect $(3 \mathrm{c})$ ou indirect libre (3d).

Il y a également plusieurs approches théoriques concernant la citation. Nous adoptons ici la théorie de la citation comme imitation de Clark et Gerrig (1990). Contrairement à Clark et Gerrig cependant, nous adoptons la deuxième définition de la citation, excluant, en particulier, le discours indirect du champ de la citation. Ainsi, sont des citations les phrases qui sont l'hôte de l'incise en (1), et les expressions entre guillemets en (3a) et (3b) ; mais ni les exemples (2) ni (3c,d) ne comportent de citation. En outre, nous spécialisons le terme de discours direct pour la construction $(3 \mathrm{a})$, où une citation est explicitement introduite par un verbe de « dire ».

Nous commençons dans la section 2 par décrire les propriétés spécifiques des incises de citation parmi les incises en général. Dans la section 3, nous discutons la nature de la citation, et précisons en quoi les incises se différencient du discours direct. La section 4 esquisse une analyse formalisée des incises de citation dans le cadre d'une grammaire HPSG (Pollard et Sag, 1994).

\section{Les incises de citation et les incises ordinaires}

\subsection{Propriétés communes}

Les incises de citation partagent avec les autres incises, ou incises « ordinaires », un certain nombre de propriétés : 
(i) Ce sont des phrases verbales : elles ont toujours pour tête un verbe, qui est saturé pour son sujet. L'incise est normalement attachée à une autre expression, que nous appellerons l'hôte de l'incise : en (1b), l'hôte est la phrase Marie était très joyeuse ${ }^{1}$.

(ii) Ce sont des expressions incidentes. A la suite de Bonami, Godard et Kampers-Manhe (2004), nous considérons l'incidence comme une propriété strictement prosodique de certains constituants syntaxiques, qui peut, suivant les constructions, être corrélée à des propriétés syntaxiques, sémantiques et pragmatiques diverses. Bien que la description de l'incidence soit à compléter, l'idée générale est claire (Fagyal 2002, Mertens 2004, Delais Roussarie 2005) : les incidents sont prosodiquement autonomes; optionnellement, ils sont séparés du reste de la phrase (notamment sur la frontière droite de l'incident) par une pause ou un allongement de la dernière syllabe, un contraste de $F_{0}$ sur la dernière syllabe, ou un changement global de registre.

(iii) Elles ont une certaine liberté de positionnement; elles sont exclues en position initiale d'énoncé, mais pas en tête d'une phrase non-initiale.

(4) a. *Paraît-il, le Président viendra ce soir.

b. Le Président, paraît-il, viendra ce soir.

c. Le Président viendra, paraît-il, ce soir.

d. Le Président viendra ce soir, paraît-il.

e. Le président viendra, et, semble-t-il, le ministre lui parlera.

(5) a. *«Annonce-t-il, Le Président est déjà arrivé. »

b. « Le Président, annonce-t-il, est déjà arrivé. »

c. ?« Le Président est, annonce-t-il, déjà arrivé. »

d. « Le Président est déjà arrivé», annonce-t-il.

e. «Cette décision, dit le président, est bonne. Et, ajouta-t-il, je ne reviendrai pas dessus. »

(iv) L'incise comporte un verbe dont le complément est manquant $(1,2 a)$ ou réalisé comme une forme pronominale $(2 \mathrm{~b}, 6)$.

(6) a. Carla, Paul vient de me dire ça, est un mannequin « très belle ».

b. Est-ce que les enfants, l'instit me l'a demandé, seront là le jour de la grève ?

(v) Il y a une forme d'identité (à préciser) entre la phrase hôte et l'interprétation du complément manquant ou pronominal de l'incise. Ainsi, les énoncés de (1) et (2) sont grossièrement équivalents à ceux qui sont donnés en (7).

(7) a. Il annonce que le Président est arrivé.

b. Paul se souvenait que Marie était très joyeuse .

c. Il paraît que Marie viendra ce soir.

d. Son porte-parole a annoncé que le président arriverait avec une heure de retard.

\subsection{Différences}

Les incises de citation diffèrent des incises ordinaires par les propriétés suivantes.

(i) Les incises de citation se caractérisent par la corrélation de deux propriétés. Alors que le complément dans les incises ordinaires est soit manquant soit un pronom, celui des incises de citation est toujours manquant. D'autre part, le sujet du verbe de citation est toujours inversé, du moins en français standard, alors que celui des incises ordinaires est parfois inversé (2a), mais pas toujours (8b), et qu'il ne l'est que s'il s'agit d'un clitique (8a) : en réalité, l'inversion ne se rencontre dans l'incise ordinaire qu'avec quelques formes qui semblent figées, comme paraît-il, semble-t-il. C'est pourquoi les incises de (9a,b) ne sont pas bonnes : il s'agit d'incises ordinaires, puisqu'elles comportent un complément pronominal ; or, dans la première, le sujet clitique est inversé alors qu'il ne s'agit pas d'une expression figée, et dans la seconde, le sujet SN est inversé. Les incises de citation correspondantes (où le complément manque) sont bonnes.

a. *Paul, croient ses collaborateurs, partira avant la fin de l'année. 
b. Paul, je crois, partira avant la fin de l'année.

a. *Le Président, l'annonce-t-il, est arrivé.

b. *Marie était très joyeuse, s'en souvenait Paul.

c. Le Président, annonce-t-il, est arrivé.

d. Marie était très joyeuse, se souvenait Paul.

Notons qu'il existe également en français informel ou non-standard, une incise de citation introduite par le complémenteur que, sans inversion : \%Le Président, qu'il disait, est arrivé. Nous ne l'analysons pas en détail ici, mais voir la note 6.

(ii) L'hôte des incises de citation est un signe linguistique de nature variée : ce peut être une interjection ou une onomatopée. L'hôte des incises ordinaires ne peut être ni une interjection, ni une onomatopée.

(10) a. « Hourrah!» a dit Paul.

b. « Grrr » a fait Paul.

(11) a. Le Président, Paul l'a dit, est arrivé.

b. *«Hourrah! » Paul l'a dit.

c. $*$ « Grrr » Paul l'a fait.

(iii) L'hôte des incises ordinaires est pris en charge illocutoirement par le locuteur ; en particulier les indexicaux de première personne y renvoient au locuteur. Par contre, l'hôte des incises de citation n'est pas pris en charge illocutoirement par le locuteur: les indexicaux de première personne y renvoient à l'agent dont les paroles sont rapportées.

(12) a. $\mathbf{M o n}_{i}$ frère $\mathrm{a}, \mathbf{P a u l}_{j} \mathbf{l}$ l'a dit, répondu correctement à ses ${ }_{j}$ questions.

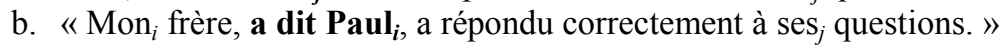

\section{$3 \quad$ L'incise de citation et les verbes de citation}

\subsection{La citation comme imitation}

Nous adoptons ici l'approche de la citation comme imitation proposée par Clark et Gerrig (1990); voir également Perrin (2002). Dans cette approche, la citation est un mode de communication qui contraste avec la description (ce qui est d'ordinaire pris en considération par les grammaires), et aussi avec la monstration (qui caractérise les déictiques, comme dans Regarde ça!). La citation est l'équivalent dans le dialogue d'un mime. Elle est caractérisée par deux propriétés : il s'agit d'un faire semblant, plutôt que d'un faire ; en (1a) le locuteur n'asserte pas, il fait semblant d'être Marie assertant l'énoncé en question. De plus, cette imitation est sélective, il ne s'agit jamais d'une reproduction intégrale, le locuteur choisissant les aspects de la situation originelle sur lesquels il veut attirer l'attention. Une citation est donc un signe imitant un signe ou un comportement produit par l'entité dénotée par le sujet du verbe de citation. Ce signe est d'une grande variété (Delaveau 1988 ; Clark et Gerrig, 1990 ; Authier-Revuz, 1992). C'est une phrase (1,3a), une interjection (13a), une onomatopée (13b), un signe non linguistique (13c), une phrase agrammaticale (13d), un segment de phrase (13e), ou une phrase comportant des auto-réparations ou du bégaiement (13f) une expression dans une autre langue (13g).

(13) a. Paul a dit : « Hourrah!»

b. Paul a fait : « Grrr».

c. Paul a fait : [le locuteur fronce les sourcils]

d. Paul a dit : « Marie est content».

e. Paul a dit : « Marie est... », mais il n'a pas pu terminer sa phrase.

f. Paul a dit: « Marie est con... con... contente ».

g. Paul a dit : «I'm hungry! ». 


\subsection{Les verbes de citation}

Les verbes susceptibles d'introduire une citation sont extrêmement variés (voir Delaveau 1988, MonvilleBurston 1993 pour les verbes de discours direct et indirect en général) : acquiescer, admettre, affirmer, annoncer, constater, demander, dénoncer, dire, faire, indiquer, lâcher, lancer, observer, ordonner, résumer, souligner, sourire, etc. Ils se groupent en trois classes en fonction de la nature de la citation. Dire introduit toute citation correspondant à un comportement linguistique. La citation ne peut pas être un comportement non-linguistique (14), mais peut prendre toutes les formes listées en (13) ci-dessus.

(14) *Paul a dit : «hips! ». (= Paul a hoqueté)

Affirmer, demander, ordonner, etc. introduisent également une citation correspondant à un comportement linguistique (15a). Celui-ci peut ne pas être un énoncé bien formé du français (15b), mais le verbe sélectionne le type d'acte de langage qui est imité : assertion (15c), interrogation ou ordre, suivant le verbe. Une question intéressante est de déterminer si la sélection se limite à l'acte effectué, ou contraint également le type de phrase utilisé pour l'effectuer. La réponse est en deux temps : quand l'imitation n'est pas en français, on ne voit pas comment la grammaire du français pourrait contraindre le type de phrase correspondant, sauf à enchâsser la grammaire de toutes les autres langues à l'intérieur de la grammaire du français. Quand l'imitation est en français, cependant, il semble bien que le type de phrase utilisé, et pas seulement l'acte de langage effectué, soit contraint. Ainsi, quand un énoncé est rituellement utilisé pour effectuer un acte de langage indirect distinct de l'acte littéral, c'est l'acte littéral qui compte pour les besoins de la citation. Ainsi, en (16), le verbe introducteur doit être demander parce que la forme de la phrase est celle d'une interrogative, bien que tout le monde sache que poser cette question équivaut pragmatiquement à 'donne-moi l'heure' ; une constatation analogue doit être faite pour (17). Cette observation suggère que dans le cas des imitations en français la sélection est une sélection grammaticalisée plutôt qu'une simple compatibilité sémantique.

(15) a. Paul a affirmé : « Marie est contente ».

b. Paul a affirmé : « Marie is happy ».

c. *Paul a affirmé : « Marie est-elle contente ?»

(16) a. *Paul a ordonné : «Est-ce que tu as l'heure ?»

b. Paul a demandé : «Est-ce que tu as l'heure ? »

a. *Paul a ordonné : « Tu roules trop vite!»

b. Paul a affirmé : «Tu roules trop vite!»

Faire introduit tout comportement, linguistique ou non. A l'oral, les comportements non-linguistiques peuvent être imités en utilisant toutes les ressources disponibles dans la situation d'énonciation (18) : parole, mime, chant, production de bruits, utilisation d'accessoires, dessin, etc. L'utilisation de faire à l'écrit présente une caractéristique remarquable: le comportement imité n'est pas nécessairement un comportement linguistique, mais si ce n'en est pas un, ce doit être un comportement sonore, imitable en utilisant l'interprétation phonétique standard de l'orthographe du français. Ainsi, l'utilisation de pictogrammes ou de dessins semble exclue dans ce contexte (19c).

(18) a. Paul a fait : [le locuteur fronce les sourcils]

b. Paul a fait : [le locuteur fredonne la Marseillaise, en chantant faux]

b. La voiture a fait : [le locuteur fait mouvement serpentant de la main]

d. La voiture a fait : [le locuteur dessine une courbe sur un papier]

(19) a. Paul a fait : « Grrr».

b. Paul a fait : « Hips!»

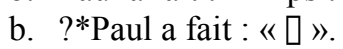

D'un point de vue syntaxique, la plupart des verbes de citation prennent normalement un complément d'objet. Mais ce n'est pas toujours le cas (Delaveau 1988) : s'exprimer, acquiescer, sourire, sont intransitifs, mais ils introduisent un adverbe de manière (ainsi), qui a le même type de dénotation. 


\subsection{Différences entre l'incise et le discours direct}

Le discours direct (3a) et l'incise de citation (1a) diffèrent par un certain nombre de points. Au delà des différences évidentes d'ordre des mots et de réalisation prosodique, il y a trois propriétés remarquables. Premièrement, les verbes concernés ne sont pas exactement les mêmes. Si tous les verbes introducteurs de discours direct sont possibles dans les incises de citation, certains verbes sont possibles dans les incises seulement ${ }^{2}$.

(20) a. «Maintenant, je me transforme en boule de feu », imagina Paul.

b. *Paul imagina : «Maintenant je me transforme en boule de feu »

Deuxièmement, alors que le discours direct se trouve sans problème dans une subordonnée (21a), la construction hôte + incise n'est pas enchâssable (21b); si enchâssement il y a, celui-ci doit avoir lieu à l'intérieur de l'incise (21c).

(21) a. Je crois que Paul a lancé à Marie : « donne-moi la main ! »

b. *Je crois que « donne-moi la main! » a lancé Paul à Marie.

c. « Donne-moi la main! », croyait avoir lancé Paul à Marie.

Troisièmement, le verbe de discours direct prend la citation comme complément (Authier-Revuz 1992), ce qui n'est pas le cas pour l'incise. Pour le discours direct, les arguments sont clairs : la citation de discours direct suit le verbe, comme le fait un complément; elle peut être enchâssée (21a); elle peut être suivie d'un autre complément, ce qui prouve qu'elle fait partie du SV (22a); elle peut être pseudo-clivée en utilisant la forme réservée aux compléments $(22 b)^{3}$. Enfin, les verbes de citation imposent des restrictions de sélection à la citation, comme noté au paragraphe 3.2. Les restrictions de sélection sont normalement imposées par un prédicat à ses arguments; quand le prédicat prend la forme d'un verbe ses arguments prennent la forme de sujet et de compléments.

(22) a. Paul a lancé « donne-moi la main ! » à Marie, avant de traverser.

b. Ce que Paul a dit, c'est « laisse-moi tranquille ! »

Dans l'incise de citation, seules les restrictions de sélection restent, ce qui motive l'analyse de l'incise, proposée dans la section 4 , comme une construction où le complément du verbe de citation est extrait et mis en relation indirecte avec la citation. Mais la citation n'a aucune des propriétés positionnelles ou fonctionnelles des compléments, comme on l'a vu plus haut: elle ne suit pas le verbe de citation (5), ne peut être encadrée par des membres du SV de l'incise (23a), ne peut être enchâssée (21b), ne peut être clivée $(23 b)$, etc.

(23) a. * * Le président », annonçait-il, « est déjà arrivé », à Marie.

b. *Ce que, annonçait-il, c’est «Le Président est déjà arrivé.»

\subsection{Différence entre l'incise de citation et l'incise de discours indirect}

La distinction entre incise de citation et incise de discours indirect (libre) n'est pas clairement reconnue dans la littérature, et elle n'est pas toujours évidente. Pour isoler l'incise de citation de l'incise ordinaire, il suffit de corréler deux propriétés, l'absence de complément et l'inversion obligatoire du sujet SN (section 2.2). Mais il existe aussi des incises qui comportent ces propriétés, avec un verbe de citation, qui ne sont pas des incises de citation : ce sont des incises de discours indirect. Il faut donc être plus précis. Différents candidats pour le discours indirect sont illustrés en (24-25).

(24) a. « Grrrr», fit Marie.

b. «I'm hungry », dit Marie.

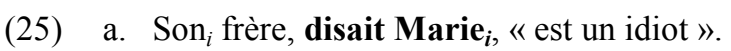

b. $\mathrm{Son}_{i}$ frère, a dit $\mathbf{M a r i e}_{i}$, ne viendrait pas avant le lendemain.

Les incises dont l'hôte n'est pas une phrase du français (24) sont des incises de citation. En effet, il est impossible de les intégrer comme discours indirect enchâssé (26). Ces incises sont nécessairement à 
droite, l'incise n'étant jamais initiale, et ce type d'expression (onomatopée, phrase dans une autre langue) ne pouvant être scindé (27).

(26) a. *Marie fit / dit que grrrr.

b. *Marie disait que she's hungry.

(27) $\quad$ a. $* « \mathrm{Gr} »$, fit Marie, « rrr ».

b. *«I'm », dit Marie, « Hungry ».

En revanche, les incises du type (25b) ne relèvent pas de la citation, mais du discours indirect. En effet, le pronom référant au sujet du verbe de citation est à la troisième personne (son frère); or, si l'on avait une citation, on aurait mon frère (comme en (29), par exemple). Plus précisément les incises de (25) sont des incises de discours indirect libre : l'hôte manifeste le type de phrase approprié pour l'acte de langage qu'il s'agit de rapporter (28), mais il n'y a pas substitution de locuteur, même par imitation. On peut proposer une analyse syntaxique identique à celle des incises de citation, à ceci près que le constituant extrait n'est pas une citation: le locuteur n'imite pas le sujet du verbe de citation, puisqu'il en parle à la troisième personne.

(28) Est-ce que son frère, se demandait Marie, viendrait avant le lendemain ?

Qu'en est-il de (25a)? Il existe des expressions citées, sans verbe introducteur, qui se comportent syntaxiquement comme une expression non citée. Elles sont mentionnées par Davidson (1979) sous le nom de « citation mixte », par Authier-Revuz (1992) sous le nom d' « îlots textuels », par Récanati (2001) sous le nom de « citation ouverte », et par Potts (2007) sous le nom de « citation de constituant».

(28) Paul me présenta son «cousin» Henri. Je savais bien qu'Henri n'était pas son cousin, mais je ne dis rien.

En (25a), la phrase hôte ne peut pas constituer en entier la citation, comme le montre le pronom son. On peut alors penser qu'on a là une incise de discours indirect dont l'hôte se trouve comporter un ilôt textuel. A partir d'exemples de ce type, il est tentant de proposer une généralisation qui lie la position de l'incise à la distinction direct/indirect : les incises de citation seraient toujours à la frontière droite de la phrase, et les incises en position médiane seraient des incises de discours indirect. Leur source ne serait pas Paul dit « $P$ » mais Paul dit que $P$. Toute citation rencontrée dans ces exemples serait un ilôt textuel enchâssé dans du discours rapporté au style indirect.

Cette généralisation ne tient pas. Premièrement, on rencontre dans des incises médianes le verbe faire (29c). Or celui-ci est toujours un verbe de citation (29a), jamais un verbe de discours indirect (29b) Deuxièmement, on a des incises médianes avec un hôte qui contient des déictiques renvoyant à l'agent du verbe de citation. Or, un déictique est difficilement inclus dans un îlot textuel $(30 \mathrm{a}, \mathrm{b})$. Un exemple comme (30c) est parfait.

(29) a. Marie ${ }_{i}$ a fait : « $\operatorname{Mon}_{i}$ frère est un idiot ».

b. *Marie a fait que son frère était un idiot.

c. « $\mathrm{Mon}_{i}$ frère », a fait Marie $i$, « est un idiot ».

(30) a. * ${ }^{P a u l}{ }_{i}$ a parlé de « $\mathrm{mon}_{i}$ frère ».

b. * $\operatorname{Son}_{i}$ frère, disait Marie $i$, « viendra demain avec $\mathrm{ma}_{i}$ nièce ».

c. " $\mathrm{Mon}_{i}$ frère, disait Marie ${ }_{i}$, viendra demain avec $\mathrm{ma}_{i}$ nièce ».

Ces observations confirment que les incises de citation peuvent bien se trouver en position médiane. On est donc amené à établir une double distinction. D'une part, les incises de citation se distinguent des incises de discours indirect: les deux types d'incises ont la même syntaxe mais n'entretiennent pas le même rapport sémantique à leur hôte. D'autre part, parmi les incises de citation, on distingue celles s'attachant à un hôte qui n'est pas une phrase du français, qui sont finales, et celles s'attachant à un hôte qui est une phrase du français, et qui peuvent être médianes ou finales. 


\section{Analyse des incises de citation}

\subsection{Analyse de la citation}

La citation est un composant de plusieurs constructions : l'incise de citation (1), le discours direct et l'îlot textuel $(3 b, c)$. Elle n'est pas caractérisable syntaxiquement. Une expression de n'importe quelle catégorie peut être le complément cité du verbe dire : Il a dit " malade / un enfant / Je serai là à $10 \mathrm{~h} /$ à $10 \mathrm{~h}$ », etc. De plus, puisque la citation peut être une expression dans une langue étrangère, lui attribuer une catégorie reviendrait à intégrer dans la grammaire du français celle d'une autre langue ! La caractérisation de la citation est dans la sémantique, et cette sémantique est très spéciale. Dans la phrase : Paul disait « Je dors ", le contenu sémantique de la citation est l'énoncé cité, pas le contenu d'une phrase (comme ce serait le cas si on avait une phrase complément). Dans les termes de Delaveau (1988, p. 133-134), « au lieu que les mots désignent des choses, ils se désignent eux-mêmes » (voir aussi Potts 2007). La combinatoire des types sémantiques est donc différente, on a, par exemple, un énoncé complet sémantiquement enchâssé. Dans notre analyse, cela est dû au mode spécifique de communication que suppose la citation, celui de l'imitation (voir section 3.1) : le constituant en mention (un énoncé en (1a)) imite le comportement originel du locuteur ou de l'agent (le sujet du verbe de citation). Cette information est présente en arrièreplan. Le contenu de la citation (c'est-à-dire l'information qu'elle apporte) est assimilé au comportement originel. Notons que c'est l'expression en mention dans son ensemble qui imite le comportement originel. Comme le note Clark et Gerrig, ce n'est pas simplement l'aspect phonologique de cette expression, qui n'est même pas toujours présent (par exemple, dans l'énoncé suivant, Marie a dit en chinois : "Le Président est arrivé », la phonologie chinoise est absente). D'un autre côté, la pragmatique est elle aussi présente, puisque le lien entre le locuteur originel et la situation originelle est reproduit (voir les indexicaux de l'expression en mention, (28)-(30)).

Nous formalisons cette analyse en utilisant une construction (31), qui part d'un énoncé ou d'un comportement du locuteur (2) et construit un nouveau signe dont le contenu (1) est un comportement (celui du locuteur ou agent originel). Une contrainte d'arrière-plan spécifie que le comportement du locuteur (2) est une imitation du comportement 1. La figure 1 illustre la citation d'un énoncé complet.

(31) La construction citation

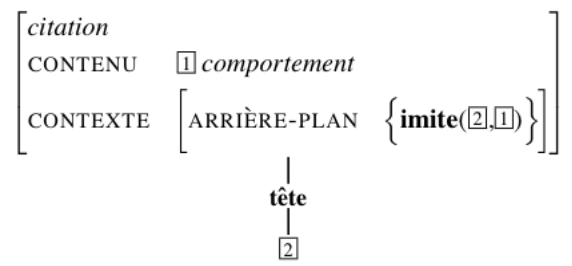

Les entrées lexicales schématiques de quelques verbes de citation sont données en (32). Ces entrées spécifient toutes que le locuteur cité coïncide avec le sujet du verbe de citation. En outre elles contraignent le type de comportement qui est cité (voir section 3.2), en s'appuyant sur une hiérarchie des comportements linguistiques et non-linguistiques que nous ne pouvons discuter en détail faute de place : un signe-linguistique est un cas particulier de comportement, une assertion est un cas particulier de signelinguistique, etc. 


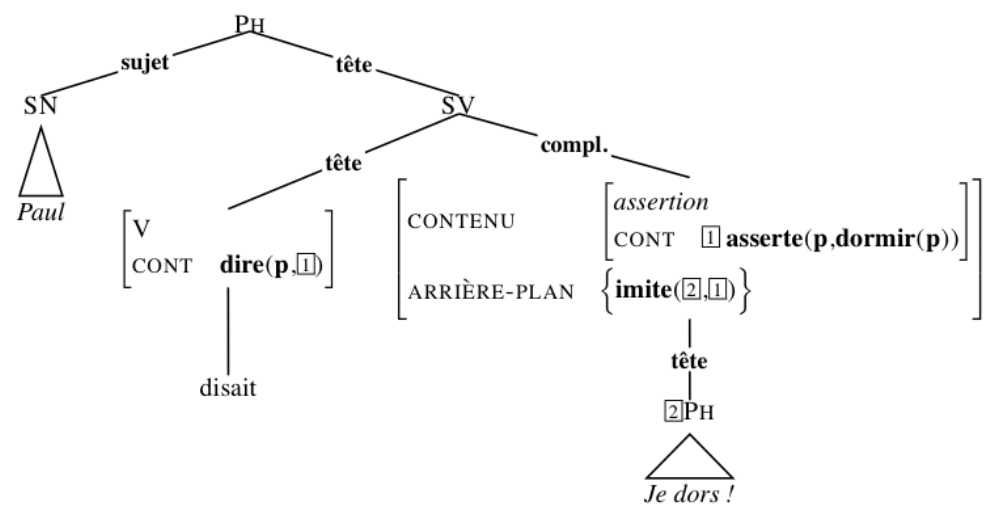

Figure 1 - Citation d'un énoncé complet

(32) a. Le verbe dire (discours direct)

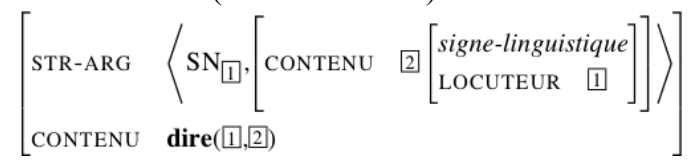

b. Le verbe affirmer (discours direct)

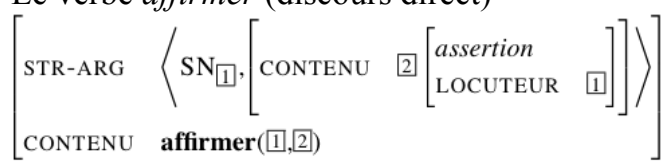

c. Le verbe faire



\subsection{Syntaxe interne de l'incise}

L'incise de citation, comme l'incise de discours indirect, est une structure à extraction : le complément manquant du verbe de citation est extrait. Les arguments sont les suivants. D'abord, il y a potentiellement une relation à distance entre le verbe de citation (dire en (33)) et le verbe supérieur de l'incise, bien qu'il y ait des restrictions :

(33) a. « Je n'en peux plus », semblait dire Paul / semblait-il dire.

b. « Je n'en peux plus », voulait dire Paul / voulait-il dire.

c. «Je n'en peux plus », semblait vouloir dire Paul / semblait-il vouloir dire.

d. « Je n'en peux plus », semblait croire pouvoir dire Paul / semblait-il croire pouvoir dire.

e. ??« Je n'en peux plus », prétendait-il que Paul avait dit.

Ensuite, cela rend compte du caractère obligatoire de l'absence du complément. Enfin, l'une des deux inversions possibles pour le sujet, celle du SN, est caractéristique des constructions à extraction, et elles seules (Kayne, 1973; Bonami et al. 1999). On distingue trois types d'inversion du sujet SN en français (Bonami et al. 1999, Marandin 2003). Dans l'inversion dite inaccusative, le verbe est un verbe intransitif, appartenant à un ensemble restreint (par exemple, les verbes d'émergence ou de disparition, Alors, apparurent trois policiers). Au contraire, le verbe de citation est le plus souvent un verbe transitif (dire, affirmer etc., voir section 3.2). Dans l'inversion élaborative, le verbe peut bien être transitif, mais le sujet doit suivre tout le SV, compléments compris (Pourront participer aux épreuves finales les étudiants 
suivants vs *Pourront participer les étudiants suivants aux épreuves finales). Dans l'inversion propre aux contextes d'extraction (dite aussi stylistique), le sujet peut se trouver entre le verbe et l'un de ses compléments. C'est ce qu'on voit avec une relative en (34a), et dans une incise de citation en (34b).

(34) a. le livre qu'a donné Marie à son fils pour son anniversaire

b. "Va te coucher », disait la mère épuisée à son fils.

Les deux dernières propriétés montrent clairement qu'il ne s'agit pas d'une structure avec ellipse, où le complément du verbe de citation serait récupéré du contexte. En effet, une ellipse n'a pas de caractère obligatoire, en général. Et surtout, ce type d'inversion du sujet $\mathrm{SN}$ ne se trouve que dans les constructions qui comportent une extraction.

Dans l'analyse de l'inversion de Bonami et al., on distingue entre la structure en constituants et leur linéarisation dans un domaine d'ordre (Reape 1994). Un mot ou un syntagme est associé à un domaine d'ordre. D'une manière générale, le domaine d'ordre d'un syntagme avec tête est une liste formée de tous ses constituants et ce domaine est compacté (considéré comme un seul élément) lorsque ce syntagme est inséré comme constituant d'un autre syntagme. Ainsi, un SV infinitif complément d'un verbe (comme dire la vérité dans Paul [semble [dire la vérité]]) compte normalement pour un seul élément dans le domaine du verbe semble. Mais, dans certaines conditions, le domaine de ce SV infinitif peut être fondu dans celui de la phrase supérieure. C'est ce qui se passe dans les structures à extraction : un SV infinitif dont le sujet est 'contrôlé' par le sujet du verbe qui le sous-catégorise, peut faire partie du même domaine d'ordre que la phrase supérieure, si son verbe est sur le trajet d'une dépendance à distance. Cela autorise alors l'inversion du sujet $\mathrm{SN}$ : le sujet de la phrase apparaît après le verbe, ou après une séquence de ce verbe et de verbes infinitifs. En (33d), l'expression semblait croire pouvoir dire Paul forme un seul domaine d'ordre, sans que la constituance soit pour cela abolie.

Formellement, un constituant extrait donne lieu à une valeur pour le trait SLASH, transmise au prédicat qui le sous-catégorise, aux syntagmes et aux prédicats dominants, rendant compte de la dépendance à distance (Ginzburg et Sag 2000) : le SLASH du complément du verbe de citation atteint le verbe supérieur de l'incise, quel que soit le degré d'enchâssement. Le domaine d'ordre est noté par le trait DOM, dont la valeur est la liste des signes formant le domaine en question. Cette liste est soumise aux contraintes d'ordre. La Figure 2 montre comment les constituants du SV inférieur ([dire] [à Marie]) de l'incise de citation sont traités comme deux éléments par le DOM du SV supérieur, et non pas comme un seul (comme le serait un SV compacté). Ce fonctionnement est illustré dans la figure 2.

A côté de l'inversion du sujet SN, l'incise de citation admet aussi l'inversion du sujet clitique (33). Celle-ci n'est pas dépendante de l'extraction d'une manière générale, mais elle est compatible avec elle (voir l'interrogative directe, Qui verrez-vous ?). A la suite de Miller (1992), nous tenons que le clitique à lui seul ne correspond pas à un signe : c'est un affixe, qui fait partie du verbe. Un verbe avec clitique sujet inversé est une forme qui fait partie du paradigme morphologique verbal en français. Comme l'inversion du SN et l'inversion affixale ont en commun de faire que le verbe apparaisse comme premier élément de l'incise, nous avançons que la valeur de DOM de l'incise obéit à la contrainte d'ordre (35), qui impose que le premier élément du domaine soit le verbe ${ }^{5,6}$.

$$
\text { incise-de-citation } \rightarrow[\mathrm{DOM}\langle[\mathrm{verbe}], \ldots\rangle]
$$




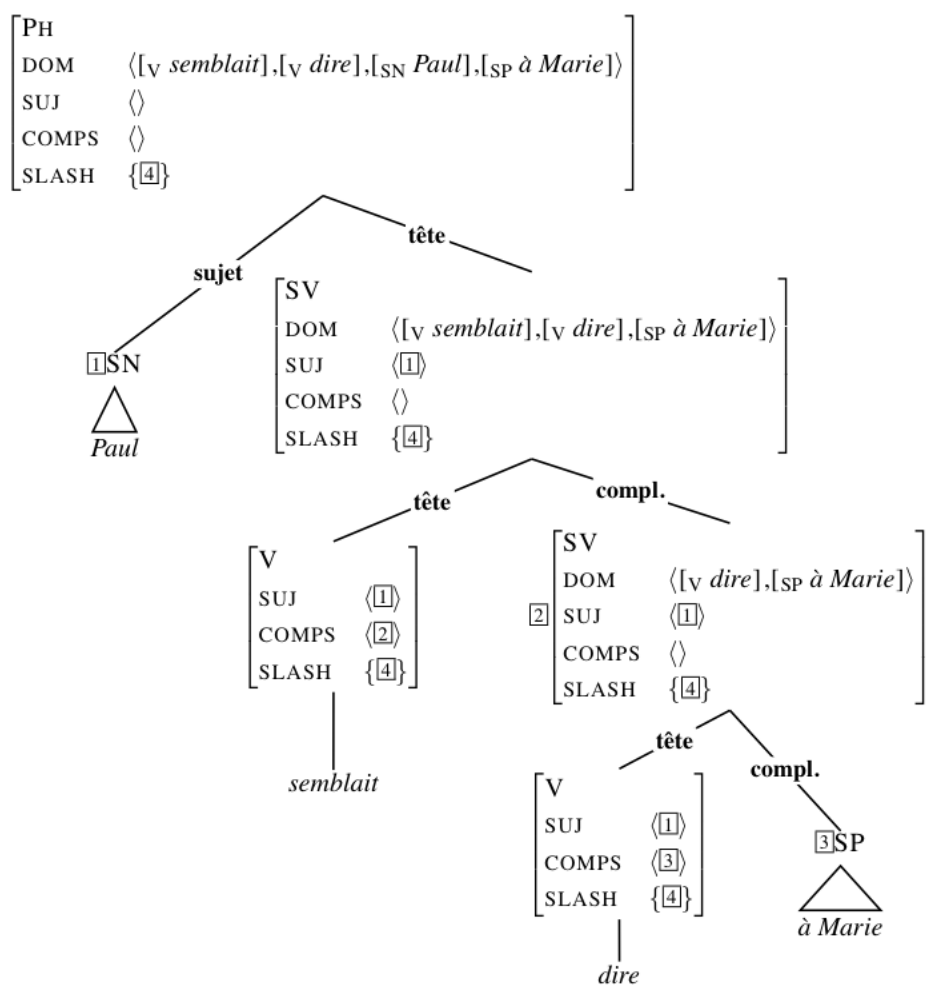

Figure 2 - Une incise à SN sujet

\subsection{La relation incise-hôte}

Voyons maintenant comment l'incise de citation se combine avec son hôte, la phrase citée. Comme pour l'ensemble des phrases incises, il s'agit d'une combinatoire de type tête-ajout, où l'incise est l'ajout. En HPSG, l'ajout sélectionne des propriétés de la tête grâce au trait MOD, dont la valeur est identifiée aux propriétés syntaxiques et sémantiques de la tête (36).

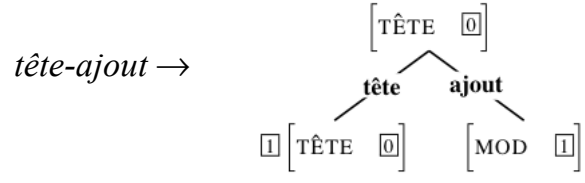

L'ajout incise peut donc sélectionner comme tête une citation. De plus, grâce au trait SLASH, dont la valeur correspond au complément extrait du verbe de citation inclus dans l'incise, nous établissons entre ce verbe et la citation (précisée dans la valeur de MOD) un rapport analogue à celui que nous avons vu entre le verbe de citation et son complément dans le discours direct. Le contenu du complément extrait est identifié à celui de la citation par la contrainte (37), comme il l'est pour les verbes de discours direct en (32). La construction arrête le trait SLASH de l'incise (voir le trait [SLASH \{\}$]$ ). 


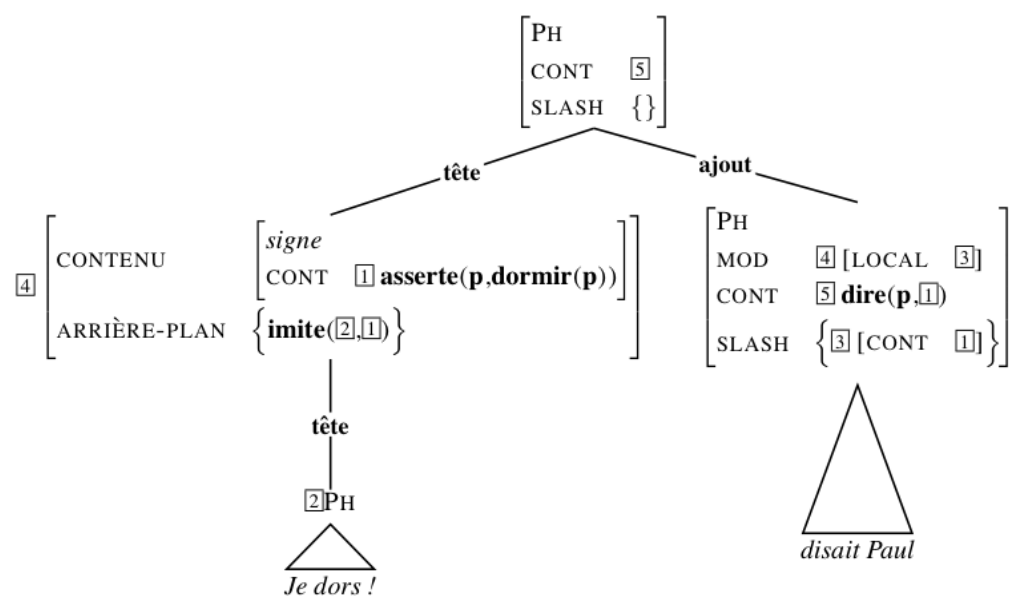

Figure 3 - Relation d'une incise de citation à son hôte

tête-incise $\rightarrow$ tête-ajout \&

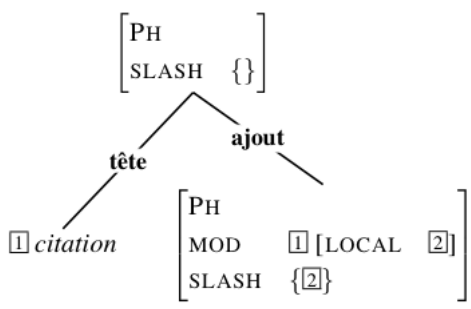

D'autre part, la construction tête-ajout proposée explique pourquoi cette construction dans son ensemble ne peut pas être enchâssée (21). La tête sémantique étant la citation, elle ne peut pas correspondre aux propriétés requises pour son argument par un prédicat ou un complémenteur : leur argument peut être une phrase d'un certain type, associé à un certain type de contenu ; par exemple, le complément de se demander est une interrogative, dont le contenu est une question (Ginzburg et Sag 2000). Mais un prédicat (sauf un verbe de citation) n'admet pas comme argument une expression dont le contenu est un énoncé.

De manière intéressante, il n'est pas nécessaire de postuler que l'incise de citation contient un verbe de type sémantique approprié : les contraintes (31) et (37) assurent que l'élément extrait dans la citation doit avoir un contenu de type comportement, ce qui est tout à fait exceptionnel : les seuls verbes qui dénotent un prédicat prenant pour argument un comportement (plutôt qu'un objet sémantique ordinaire) sont les verbes de citation. Un exemple de construction avec incise de citation est donné dans la Figure 3.

\subsection{Les incises insérées : une analyse par linéarisation}

Comme nous l'avons vu (5), l'incise de citation jouit d'une certaine liberté de positionnement à l'intérieur de la phrase hôte. Dans une approche qui fait appel aux domaines d'ordre et aux contraintes de linéarisation (voir section 4.2), cela veut dire que l'incise est un élément du domaine d'ordre de la phrase tête, et qu'elle est peu contrainte. Revenons à la relation entre les constituants et les domaines d'ordre. Nous admettons, à la suite de Kathol (2000), que le domaine d'un syntagme est constitué de sa tête avec tous ses valents et ajouts. Autrement dit, comme indiqué en (38), la tête n'est jamais compactée, alors que les constituants non-têtes sont généralement compactés-le cas des phrases à inversion faisant exception. 


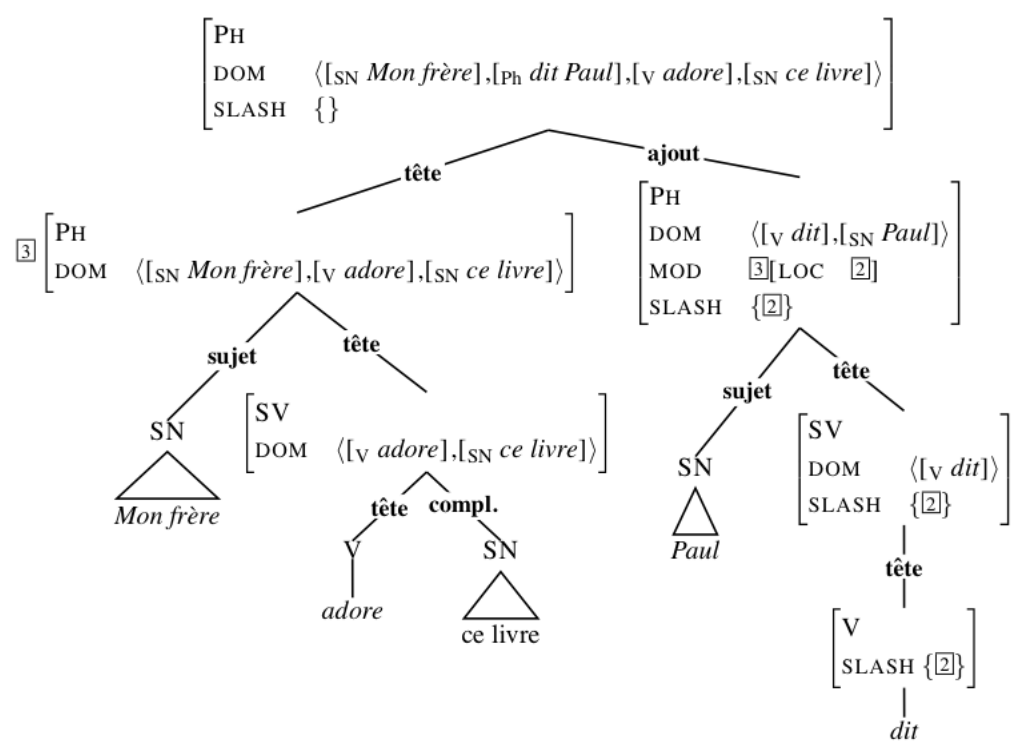

Figure 4 - Une incise insérée

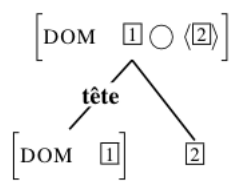

C'est donc en tant qu'ajout que l'incise de citation peut être intégrée à la phrase hôte : comme tous les ajouts, l'incise est un dépendant du verbe, et comme tous les ajouts, elle se place relativement librement par rapport à ce verbe ; elle a précisément la distribution des ajouts incidents (voir, pour les adverbes incidents, Bonami et Godard 2007). Les incises cependant obéissent à une contrainte d'ordre, illustrée en (4)-(5) : elles n'apparaissent pas en début d'énoncé. La contrainte (39) empêche l'incise de citation d'apparaître comme premier élément du domaine d'ordre d'un énoncé. L'incise de citation est répérable ici par la propriété suivante : un complément a été extrait, dont le contenu est un comportement.

(39) énoncé $\rightarrow[\operatorname{DOM}\langle\neg[\mathrm{SLASH}\{[\mathrm{CONT}$ comportement $], \ldots\}] \ldots\rangle]$

\section{Références bibliographiques}

Authier-Revuz, J. (1992-93) : Repères dans le champ du discours rapporté. L'information Grammaticale 55, 38-42, et $56,10-15$.

Bonami, O. et Godard, D. (2007). Integrating linguistic dimensions: The scope of adverbs. In S. Muller (ed.), Proceedings of the 14th International Conference on Head-driven Phrase Structure Grammar. Stanford : CSLI Publictaions (on line), 25-45. 
Bonami, O., Godard, D., et Marandin, J.-M. (1999). Constituency and word order in French subject inversion ». In Bouma, G., Hinrichs, E., Kruijff, G.-J., et Oerhle, R. (éd.), Constraints and Resources in Natural Language Syntax and Semantics, Stanford : CSLI Publications, p. 21-40.

Bonami, O., Kampers-Manhe, B., et Godard, D. (2004). Adverb Classification. In Corblin, F. et de Swart, H. (éd.), Handbook of French Semantics, Stanford : CSLI Publications, p. 143-184.

Clark, H.H. et Gerrig, R. J. (1990). Quotations as demonstrations. Language 66, 764-805.

Davidson, D. (1979). Quotation. Theory and decision, 11, 27-40. (repris dans Inquiries into Truth and Interpretation, Oxford : Oxford University Press, 1984).

Delais-Roussarie, E., (2005). Vers une Grammaire prosodique formelle : le cas des incidentes en français ». In Actes électroniques de la conférence Interface Discours et Prosodie 05, Université de Provence.

Delaveau, A. (1988). La Voix et les bruits. Note sur les verbes introducteurs du discours rapporté. LINX 18, 125-135.

Fagyal, Z. (2002). Prosodic boundaries in the vicinity of utterance-medial parentheticals in French. Probus 14, 93111.

Ginzburg, J. et Sag, I. A. (2000). Interrogative Investigations. Stanford : CSLI Publications.

Kathol, A. (2000). Linear Syntax. Oxford : Oxford University Press.

Kayne, R. (1973). L'inversion du sujet en français dans les propositions interrogatives. Le français moderne, 41, 1042.

Marandin, J.-M. (2003). Inversion du sujet et discours dans les langues romanes. In D. Godard (ed.), Les Langues Romanes. Paris : CNRS Editions, 345-392.

Mertens, P. (2004). Quelques aller-retour entre la prosodie et son traitement automatique. Le français moderne 72 , 39-57.

Miller, P. (1992). Clitics and Constituents in Phrase Structure Grammar. New York : Garland.

Monville Burston, M. (1993). Les verba dicendi dans la presse d'information. Langue Française 98, 48-66.

Perrin, L. (2002). Les formes de la citation au style direct, indirect et indirect libre. Faits de langue 94, 147-158.

Pollard, C. et Sag, I. A. (1994). Head Driven Phrase Structure Grammar. Stanford : CSLI Publications et Chicago : The University of Chicago Press.

Potts, C. (2007). The Dimensions of Quotation. In C. Barker and P. Jacobson (eds), Direct Compositionality. Oxford : Oxford University Press, 405-431.

Reape, M. (1994). Domain union and word order variation in German. In Nerbonne, J., Netter, K. et Pollard, C. (éds.), German in HPSG, Stanford : CSLI Publications, 151-197.

Récanati, F. (2001). Open Quotation. Mind 110, 637-687.

\footnotetext{
* Nous remercions Elisabeth Richard, Frank Richter, Manfred Sailer et Jan-Philipp Söhn, ainsi qu'un relecteur anonyme, pour leurs commentaires et suggestions. Cette recherche s'inscrit dans le cadre de l'axe constructions incidentes du projet ANR PRO-GRAM (http://pro-gram.linguist.jussieu.fr).

${ }^{1}$ En général, les incises ne fonctionnent pas comme des énoncés autonomes ; comme la plupart des constituants elles peuvent cependant constituer une réponse dans un dialogue, l'hôte n'étant pas repris (i). Elles peuvent également être homonymes d'énoncés autonomes si le complément est pronominal, comme en (7).
}

(i) A : Paul est arrivé ? - B : Il paraît.

${ }^{2}$ Ces verbes tendent à être des verbes de parole par extension métaphorique (Delaveau 1988). Ainsi, imaginer est assimilé à 'se dire à soi-même'. On prendra garde, cependant, à distinguer entre verbes de citation et verbes de discours indirect (libre), voir section 3.4. Ainsi, montrer, estimer sont seulement des verbes de discours indirect.

(i) Cette hypothèse, voulait montrer Paul / estimait Paul, est celle qui tient le mieux la route.

(ii) $* \mathrm{Mon}_{i}$ hypothèse, voulait montrer $\mathrm{Paul}_{i} /$ estimait $\mathrm{Paul}_{i}$, est celle qui tient le mieux la route. 


\footnotetext{
${ }^{3}$ Nous traitons les expressions de manière comme des compléments du verbe. Ainsi, lorsque le verbe n'attend pas de complément d'objet (comme s'exclame, s'écrier), mais un adverbe de manière, la citation a encore la fonction de complément (de complément oblique plutôt que de complément d'objet).

${ }^{4}$ La phrase (29b) est bonne avec une autre interprétation (où faire que = « faire en sorte que »).

${ }^{5}$ On pourrait penser que la contrainte vise directement l'inversion. Mais cela ne suffirait pas à éliminer la possibilité d'une incise comme *Je viendrai, Paul disait-il, où le verbe est bien inversé (la forme est possible dans l'interrogative directe).

${ }^{6}$ On sait que, dans un français moins formel, les incises de citation peuvent prendre la forme d'une phrase introduite par que, sans inversion (i).
}

(i) \%Alors, qu'il me dit, quand est-ce qu'on part?

Ces incises sont semblables aux relatives en que : leurs têtes sont des complémenteurs, et elles comportent un constituant extrait ; mais, au lieu que le SLASH soit arrêté par la construction dans son ensemble (comme pour l'incise à inversion, (35)), il est arrêté par le complémenteur. La différence essentielle, bien sûr, concerne la tête à laquelle la phrase s'adjoint. 